\title{
What Event-Related Potential Tells Us about Brain Function: Child-Adolescent Psychiatric Perspectives
}

\author{
Ji Sun Kim¹, Yeon Jung Lee ${ }^{2}$, and Se-Hoon Shim ${ }^{1}$ \\ ${ }^{1}$ Department of Psychiatry, Soonchunhyang University Cheonan Hospital, Soonchunhyang University College of Medicine, Cheonan, Korea \\ ${ }^{2}$ Department of Psychiatry, Soonchunhyang University Seoul Hospital, Soonchunhyang University College of Medicine, Seoul, Korea
}

\begin{abstract}
Electroencephalography (EEG) measures neural activation due to various cognitive processes. EEG and event-related potentials (ERPs) are widely used in studies investigating psychopathology and neural substrates of psychiatric diseases in children and adolescents. The present study aimed to review recent ERP studies in child and adolescent psychiatry. ERPs are non-invasive methods for studying synaptic functions in the brain. ERP might be a candidate biomarker in child-adolescent psychiatry, considering its ability to reflect cognitive and behavioral functions in humans. For the EEG study of psychiatric diseases in children and adolescents, several ERP components have been used, such as mismatch negativity, P300, error-related negativity (ERN), and reward positivity (RewP). Regarding executive functions and inhibition in patients with attention-deficit/hyperactivity disorder (ADHD), P300 latency, and ERN were significantly different in patients with ADHD compared to those in the healthy population. ERN showed meaningful changes in patients with anxiety disorders, such as generalized anxiety disorder, separation anxiety disorder, and obsessive-compulsive disorder. Patients with depression showed significantly attenuated RewP compared to the healthy population, which was related to the symptoms of anhedonia.
\end{abstract}

Key Words: Electroencephalography; Event-related potentials; Child psychiatry; Psychopathology.

Received: April 23, 2021 / Revision: May 13, 2021 / Accepted: May 21, 2021

Address for correspondence: Se-Hoon Shim, Department of Psychiatry, Soonchunhyang University Cheonan Hospital, Soonchunhyang University College of Medicine, 31 Suncheonhyang 6-gil, Dongnam-gu, Cheonan 31151, Korea Tel: +82-41-570-3853, Fax: +82-41-592-3804, E-mail: shshim2k@daum.net

\section{INTRODUCTION}

Electroencephalography (EEG) allows the non-invasive assessment of cognitive processes in real time [1]. The superior time resolution of EEG can follow fast neural events and permit the knowledge of related brain regions through source analysis [1]. It can directly assess the dynamics of neural activation related to various cognitive processes [2]. Moreover, it can be assessed in infants and children [3]; therefore, EEG was the first tool used to assess neural activities in children [4], and event-related potentials (ERPs) have been used in various studies to investigate psychopathology and neural substrates of psychiatric diseases in children $[1,4]$.

This review aims to explain the current body of ERP studies on psychiatric illnesses in children and adolescents. It is important to understand how ERP studies have played a role in enhancing our understanding of neural functions in childhood and adolescence. This review starts with an introduction to the use of EEG and ERP to assess child-adolescent psychiatric diseases. Second, the definition of ERP and various

This is an Open Access article distributed under the terms of the Creative Commons Attribution Non-Commercial License (https://creativecommons.org/licenses/by-nc/4.0) which permits unrestricted non-commercial use, distribution, and reproduction in any medium, provided the original work is properly cited.
ERP markers for child-adolescent psychiatric diseases will be outlined. Finally, recent advances in ERP research on representative child-adolescent psychiatric diseases will be reviewed.

\section{Definitions of ERP}

ERPs are scalp-recorded voltage changes that measure the response of the brain to a time-locked event [5]. In other words, ERPs allow us to determine the activities of coordinated neurons assessed by averaging sections of electrical recordings that are time-locked to motor, perceptual, and cognitive events [6]. The timing, size, and location of ERPs on the scalp infer the time course of neural processing in the brain [6]. Therefore, ERPs reflect thousands of simultaneously ongoing brain processes that can be used to evaluate perceptual processing, sensory-motor coupling, and cognition, such as attentional selection [7].

\section{Use of EEG and ERP to assess child-adolescent psychiatric disease}

ERPs enable a temporally detailed exploration of the cognitive process; early components of ERP reflect sensory or perceptual processing, and later components reflect higher 
cognitive processes [8]. In particular, cognitive ERPs provide a powerful method for investigating synaptic functions in the brain. ERPs reflect excitatory postsynaptic potentials (EPSPs) and inhibitory postsynaptic potentials (IPSPs) [9-11]. Because ERP allows us to know the temporal patterns of neuronal activities, it can be useful in quantifying the sequence of various cognitive processes [12]. Considering that children and adolescents are in the phase of development, research involving ERPs will allow us to understand the association between brain development and behavioral development, which might provide important evidence [3].

There are many applications of EEG and ERP in child-adolescent psychopathologies within the context of biomarkers of disease [4]. Child-adolescent psychiatric illnesses are related to various factors, such as developmental course, clinically heterogeneous etiology, clinical manifestation, and treatment response. Therefore, the identification of biomarkers to facilitate diagnosis and predict treatment response is critical in this field. ERP could be a candidate biomarker in child-adolescent psychiatry, considering its ability to reflect cognitive and behavioral functions in humans.

From a practical perspective, it is crucial to use simple paradigms, especially for children. The stimuli for the ERP were relatively simple, such as a three-tone (standard, deviant, novel) auditory oddball paradigm [13]. Moreover, EEG and ERPs are non-invasive and inexpensive assessment methods; thus, they are useful for both clinical evaluation and research involving adolescents or children. Furthermore, the ERP is less affected by movement artifacts than other brain imaging methods, and it could be useful for studying children.

\section{Various ERP markers for child-adolescent psychiatric diseases}

\section{MMN}

Mismatch negativity (MMN) is an ERP component when a sequence of relatively standard stimuli is interrupted by the infrequent presentation of deviant stimuli [14]. In addition, MMN reflects preattentive auditory processing, which is closely correlated with cognitive status $[15,16]$. Regarding patients with psychiatric disease experiencing cognitive dysfunction, MMN has been considered a good tool for evaluating the neurophysiological mechanisms of psychiatric illness. Moreover, MMN reflects glutamatergic function [17], and the critical pathology of MMN reduction might be related to dysfunction of the N-methyl-D-aspartate (NMDA) receptor system [18]. NMDA receptors have been studied for their essential role in cognitive functions, such as learning and memory [19]. In this regard, $\mathrm{MMN}$ is considered a promising biomarker for many psychiatric disorders.

\section{P300, Go/NoGo paradigm, and Flanker task}

The P300 (P3) wave has two components, P3a and P3b. The $\mathrm{P} 3 \mathrm{a}$ is a positive wave that appears maximally in the fronto-central region and has a peak latency of 250-280 ms [20]. It reflects the brain electrical activity related to decision-making, attention, and novelty processing [20]. The $\mathrm{P} 3 \mathrm{~b}$ component has a positive amplitude that peaks at approximately $300 \mathrm{~ms}$; the peak will vary in latency from $250 \mathrm{~ms}$ to $500 \mathrm{~ms}$ or more. $\mathrm{P} 3$ was evoked using the oddball paradigm in which the target stimulus was mixed with a non-target stimulus. In particular, $\mathrm{P} 3$ can be elicited using various tasks, such as $\mathrm{Go} /$ NoGo and Flanker tasks. The Go/Nogo paradigm reflects behavioral and response inhibition [21,22]. NogoP3 is a wellknown indicator of behavioral control [23]. Specifically, P3 reflects motor inhibition [24]

\section{ERN}

Error-related negativity (ERN) is a sharp negative amplitude that begins around the same time as an incorrect motor response [25], which occurs when a person makes an error [26]. It is evoked using cognitive tasks when a person has an incorrect response [27], and it appears as a negative wave at $80-150 \mathrm{~ms}$ following error commission [27]. Pe is a positive wave that appears subsequent to ERN and is the highest at $300 \mathrm{~ms}$ post-incorrect response [26]. Although both Pe and ERN are associated with the error-related response, they are different in that $\mathrm{Pe}$ is related to post-error possessing [28], and ERN reflects the comparison process between an actual response and a required response [29]. That is, ERN can arise even after unaware errors, while Pe reflects conscious error processing [29]. Because ERN and Pe have different aspects of error processing, the two components have different neural generators [29]. Source localization indicates that ERN has a dipole in the anterior cingulate cortex, and Pe has a dipole in the posterior cingulate cortex [30].

\section{RewP}

Reward positivity (RewP) reflects reward responsiveness [31]. RewP is a positive wave following positive information and is either decreased or absent when obtaining negative information $[32,33]$. In other words, sensitivity to reward or nonreward stimuli can be represented by RewP [34]. RewP is the highest in medial frontocentral areas and appears at 250-550 ms post reward [31]. The amplitude of the RewP also predicts individual differences in sensitivity to reward, as assessed by both self-report and behavioral measures [35,36]. 


\section{ERP markers for child-adolescent psychiatric disease}

\section{ADHD}

Previous studies have discussed P3 latency in children with attention-deficit/hyperactivity disorder (ADHD). Participants with ADHD had an increased visual P3 latency and decreased P3 amplitude compared to healthy participants, suggesting reduced involvement in post-decisional processing [37]. Latency reflects attention, regarding it is various to the effort of discriminating different stimuli [38]. Latency is also associated with stimulus evaluation and discrimination [39]. Moreover, children with ADHD show increased latency and stimulants (methylphenidate) have been found to reduce the latency [38]. A previous study pointed out that children with ADHD have prolonged P3 latency at the frontocentral electrodes, and the level of prolongation also has a positive correlation with the severity of inattention [40]. This suggests that P3 latency is a neurophysiological marker in ADHD and may change under stimulant treatment [38].

Executive dysfunction is one of the well-known etiologies of ADHD, resulting in reduced cognitive function and abnormal behavior $[41,42]$. One of the most prominent etiological theories explains executive dysfunction as a secondary clinical manifestation of deficits in behavioral inhibition $[43,44]$. Specifically, the role of inhibition in ADHD has been emphasized by Nigg; the author insisted that ADHD is associated with deficits in behavioral inhibition [44-46]. On the other hand, error monitoring can show the self-monitoring of a person's own behavior, and it can make people assess their own response considering the actual demand. Therefore, it can recognize a potential error and help people adjust their response to prevent additional errors [44-46]. Patients with ADHD make many careless mistakes, and it could be expected that cognitive dysfunctions underlying error monitoring in various situations that require highly intended and about-to-be-performed responses may exist [44,45]. In this regard, error monitoring can be assessed using various tasks such as the Go/NoGo paradigm, stop-signal task, and Flanker task.

For the Go/NoGo paradigm, several previous studies reported that juvenile patients with ADHD have significantly different ERN compared to the healthy population [47-49]. In studies that used the Flanker task, both the Pe and Ne/ERN were decreased in patients with ADHD compared with the healthy population, and this finding was pronounced in the younger population [50].

One longitudinal EEG study that explored the course of impaired cognitive functions in ADHD has shown interesting results; all ERP components such as Cue P3, NoGo P3, and contingent negative variation $(\mathrm{CNV})$ developed without significant time $\times$ group interactions [51]. Only CNV remained decreased in the ADHD group from childhood to adulthood [51]. This suggests that attentional and preparatory deficits in ADHD continue into adulthood, and attenuated CNV reflects a particularly stable ADHD marker. Further longitudinal studies are required to confirm these results [51].

\section{Anxiety disorder}

Anxiety disorder is one of the most common forms of childadolescent psychopathologies and often begins early in human development [52]. ERN has been studied extensively in patients with anxiety disorders during childhood and adolescence. The ERN was shown to be increased in children with anxiety using various experimental paradigms to assess ERN $[6,52,53]$. Similar results were obtained using Flanker $[6,52,53]$ and Go/NoGo tasks [54]. Ladouceur et al. [6] reported that children with anxiety disorder had increased ERN. In addition, a large ERN early in life (childhood and adolescence) was associated with an increased risk of developing anxiety disorder later in life [6,53]. Previous studies found that a larger ERN predicted the onset of anxiety disorder, while controlling for baseline anxiety symptoms and maternal history of anxiety $[6,53,55]$.

A previous study that evaluated behavioral performance using the Go/NoGo task in a sample of 139 children aged 5-8 years reported that symptoms of separation anxiety disorder (SAD) were related to a small ERN, even after controlling for other anxiety disorder symptoms [56]. Additionally, children with more SAD symptoms showed higher error rates and failed to exhibit the expected association between behavioral performance and ERN, suggesting the presence of ineffective error-monitoring in children with SAD [56].

A recent prospective study showed meaningful results for the development of generalized anxiety disorder (GAD) [57] in 457 girls aged between 13.5 years and 15.5 years, with no history of GAD. The study revealed that the ERN using the Flanker task was a significant predictor independent of other risk factors, such as baseline anxiety and depression symptoms, and parental lifetime psychiatric history. This suggests the utility of ERN as a biomarker of GAD risk during a key developmental period.

Additionally, Carrasco et al. [53] revealed that the ERN magnitude was associated with obsessive-compulsive disorder (OCD) symptoms in children and adolescents without clinical OCD. This study reported that ERN was increased in unaffected siblings of children with OCD [53]. Moreover, a recent study found an increased ERN in children after successful treatment for OCD [58]. These studies suggest that children at risk for OCD may be characterized by increased ERN. 


\section{Mood disorders}

Decreased motivation and reactivity toward pleasurable stimuli are the usual clinical manifestations of depression [59]. Depression onset often occurs in adolescence, the peak period for neural development of reward circuitry $[42,60]$. Blunted neural responses to RewP are related to vulnerability to depression [34]. A previous study with a community sample of 369 children showed that stressful life events moderated the effect of RewP on depression symptoms at follow-up [33]. In other words, blunted RewP predicted high risk of depression symptoms in individuals with high levels of stressful life events [33]. Another study with 20 adolescents aged 12-17 years reported direct effects of the RewP latency on depression symptoms and withdrawal behaviors [34]. In addition, longer reward latency is associated with stronger depression [34]. This suggests that avoidance motivation can help demonstrate the association between reduced reward responsivity and symptoms of depression [34].

\section{CONCLUSION}

Investigating ERPs is a noninvasive method to assess spatio-temporal activation in the brain during sensory, cognitive, affective, attentional, and motor information processing as well as during state regulation in real time. They can reliably be reproduced in a wide range of patients with psychiatric disease and across a wide age range, even in infants and children, and during both wakefulness and sleep. ERPs can be an ideal tool for studies of brain function during normal and deviant child development. Although more evidence related to various psychiatric conditions is needed, ERPs could be a candidate biomarker for studying child-adolescent psychiatry, considering their ability to reflect cognitive and behavioral functions in humans.

\section{Acknowledgments}

This work was supported by a grant (2020R1I1A3068017) awarded by the Basic Science Research Program through the National Research Foundation of Korea (NRF) and funded by the Ministry of Education. This work was also supported by a grant (2020R1I1A3A04036435) awarded by the Basic Science Research Program through the National Research Foundation of Korea (NRF) and funded by the Ministry of Education.

\section{Conflicts of Interest}

The authors have no potential conflicts of interest to disclose.

\section{Author Contributions}

Conceptualization: Ji Sun Kim. Funding acquisition: Ji Sun Kim, Se-Hoon Shim. Investigation: Ji Sun Kim, Yeon Jung Lee. Methodology: Ji Sun Kim, Yeon Jung Lee. Project administration: Ji Sun Kim, Se-Hoon Shim. Resources: Ji Sun Kim, Se-Hoon Shim. Software: Ji Sun Kim, Yeon Jung Lee. Supervision: Se-Hoon Shim. Writing_-original draft: Ji Sun Kim, Yeon Jung Lee. Writing_-review \& editing: Ji Sun Kim, Se-Hoon Shim.

\section{ORCID iDs}

Ji Sun Kim

https://orcid.org/0000-0003-2472-4591

Yeon Jung Lee

https://orcid.org/0000-0001-8953-5893

Se-Hoon Shim

https://orcid.org/0000-0002-3137-6591

\section{REFERENCES}

1) Banaschewski T, Brandeis D. Annotation: what electrical brain activity tells us about brain function that other techniques cannot tell us-a child psychiatric perspective. J Child Psychol Psychiatry 2007; 48:415-435.

2) Picton TW, Bentin S, Berg P, Donchin E, Hillyard SA, Johnson R Jr, et al. Guidelines for using human event-related potentials to study cognition: recording standards and publication criteria. Psychophysiology 2000;37:127-152.

3) Taylor MJ, Baldeweg T. Application of EEG, ERP and intracranial recordings to the investigation of cognitive functions in children. Dev Sci 2002;5:318-334.

4) Loo SK, Lenartowicz A, Makeig S. Research review: use of EEG biomarkers in child psychiatry research-current state and future directions. J Child Psychol Psychiatry 2016;57:4-17.

5) Herrmann CS, Knight RT. Mechanisms of human attention: eventrelated potentials and oscillations. Neurosci Biobehav Rev 2001;25: 465-476.

6) Ladouceur CD, Dahl RE, Birmaher B, Axelson DA, Ryan ND. Increased error-related negativity (ERN) in childhood anxiety disorders: ERP and source localization. J Child Psychol Psychiatry 2006; 47:1073-1082.

7) Al-Ezzi A, Kamel N, Faye I, Gunaseli E. Review of EEG, ERP, and brain connectivity estimators as predictive biomarkers of social anxiety disorder. Front Psychol 2020;11:730.

8) Woodman GF, Luck SJ. Serial deployment of attention during visual search. J Exp Psychol Hum Percept Perform 2003;29:121-138.

9) Nunez PL. Physical principles and neurophysiological mechanisms underlying event-related potentials. In: Rohrbaugh JW, Parasuraman R, Johnson R, Johnson SSFR, editors. Event-related brain potentials: basic issues and applications. New York: Oxford University Press;1990. p.19-36.

10) Nunez PL, Srinivasan R. Electric fields of the brain: the neurophysics of EEG. New York: Oxford University Press;2006. p.163-166.

11) Wood CC, Allison T. Interpretation of evoked potentials: a neurophysiological perspective. Can J Psychol 1981;35:113-135.

12) Olichney JM, Yang JC, Taylor J, Kutas M. Cognitive event-related potentials: biomarkers of synaptic dysfunction across the stages of Alzheimer's disease. J Alzheimers Dis 2011;26 Suppl 3:215-228.

13) Marshall PJ, Reeb BC, Fox NA. Electrophysiological responses to auditory novelty in temperamentally different 9 -month-old infants. Dev Sci 2009;12:568-582.

14) Näätänen R, Gaillard AW, Mäntysalo S. Early selective-attention effect on evoked potential reinterpreted. Acta Psychol (Amst) 1978; 42:313-329.

15) Javitt DC, Zukin SR, Heresco-Levy U, Umbricht D. Has an angel shown the way? Etiological and therapeutic implications of the PCP/NMDA model of schizophrenia. Schizophr Bull 2012;38:958966.

16) Näätänen R, Kujala T, Escera C, Baldeweg T, Kreegipuu K, Carlson $\mathrm{S}$, et al. The mismatch negativity (MMN)--a unique window to disturbed central auditory processing in ageing and different clinical conditions. Clin Neurophysiol 2012;123:424-458.

17) Chitty KM, Lagopoulos J, Lee RS, Hickie IB, Hermens DF. A systematic review and meta-analysis of proton magnetic resonance spectroscopy and mismatch negativity in bipolar disorder. Eur Neuropsychopharmacol 2013;23:1348-1363.

18) Javitt DC, Doneshka P, Grochowski S, Ritter W. Impaired mismatch negativity generation reflects widespread dysfunction of working 
memory in schizophrenia. Arch Gen Psychiatry 1995;52:550-558.

19) Lakhan SE, Caro M, Hadzimichalis N. NMDA receptor activity in neuropsychiatric disorders. Front Psychiatry 2013;4:52.

20) Polich J. Updating P300: an integrative theory of P $3 a$ and P3b. Clin Neurophysiol 2007;118:2128-2148.

21) Bokura H, Yamaguchi S, Kobayashi S. Electrophysiological correlates for response inhibition in a $\mathrm{Go} / \mathrm{NoGo}$ task. Clin Neurophysiol 2001;112:2224-2232.

22) Kim EJ, Kwon YJ, Lee HY, Yoon HJ, Kim JS, Shim SH. The relationship between response-inhibitory event-related potentials and symptoms of attention-deficit/hyperactivity disorder in adult patients with major depressive disorder. Psychiatry Investig 2020;17: 996-1005.

23) Kim JS, Kim S, Jung W, Im CH, Lee SH. Auditory evoked potential could reflect emotional sensitivity and impulsivity. Sci Rep 2016;6: 37683.

24) Enriquez-Geppert S, Konrad C, Pantev C, Huster RJ. Conflict and inhibition differentially affect the N200/P300 complex in a combined go/nogo and stop-signal task. Neuroimage 2010;51:877-887.

25) Gruendler TO, Ullsperger M, Huster RJ. Event-related potential correlates of performance-monitoring in a lateralized time-estimation task. PLoS One 2011;6:e25591.

26) Crowley MJ, Wu J, Hommer RE, South M, Molfese PJ, Fearon RM, et al. A developmental study of the feedback-related negativity from 10-17 years: age and sex effects for reward versus non-reward. Dev Neuropsychol 2013;38:595-612.

27) Speed BC, Jackson F, Nelson BD, Infantolino ZP, Hajcak G. Unpredictability increases the error-related negativity in children and adolescents. Brain Cogn 2017;119:25-31.

28) Chang YK. Acute exercise and event-related potential: current status and future prospects. In: McMorris T, editor. Exercise-cognition interaction: neuroscience perspectives. San Diego: Academic Press;2016. p.105-130.

29) Herrmann MJ, Römmler J, Ehlis AC, Heidrich A, Fallgatter AJ. Source localization (LORETA) of the error-related-negativity (ERN/ $\mathrm{Ne}$ ) and positivity (Pe). Brain Res Cogn Brain Res 2004;20:294299.

30) Ito S, Stuphorn V, Brown JW, Schall JD. Performance monitoring by the anterior cingulate cortex during saccade countermanding. Science 2003;302:120-122.

31) Proudfit GH. The reward positivity: from basic research on reward to a biomarker for depression. Psychophysiology 2015;52:449-459.

32) Nelson BD, Infantolino ZP, Klein DN, Perlman G, Kotov R, Hajcak G. Time-frequency reward-related delta prospectively predicts the development of adolescent-onset depression. Biol Psychiatry Cogn Neurosci Neuroimaging 2018;3:41-49.

33) Goldstein BL, Kessel EM, Kujawa A, Finsaas MC, Davila J, Hajcak G, et al. Stressful life events moderate the effect of neural reward responsiveness in childhood on depressive symptoms in adolescence. Psychol Med 2020;50:1548-1555.

34) Ellis AJ, Salgari G, Miklowitz DJ, Loo SK. The role of avoidance motivation in the relationship between reward sensitivity and depression symptoms in adolescents: an ERP study. Psychiatry Res 2019;279:345-349.

35) Bress JN, Hajcak G. Self-report and behavioral measures of reward sensitivity predict the feedback negativity. Psychophysiology 2013; 50:610-616.

36) Carlson JM, Foti D, Mujica-Parodi LR, Harmon-Jones E, Hajcak G. Ventral striatal and medial prefrontal BOLD activation is correlated with reward-related electrocortical activity: a combined ERP and fMRI study. Neuroimage 2011;57:1608-1616.

37) Picton TW. The P 300 wave of the human event-related potential. J Clin Neurophysiol 1992;9:456-479.

38) Chi MH, Chu CL, Lee IH, Hsieh YT, Chen KC, Chen PS, et al. Altered auditory P300 performance in parents with attention deficit hyperactivity disorder offspring. Clin Psychopharmacol Neurosci 2019;17:509-516.

39) Ozdag MF, Yorbik O, Ulas UH, Hamamcioglu K, Vural O. Effect of methylphenidate on auditory event related potential in boys with attention deficit hyperactivity disorder. Int J Pediatr Otorhinolaryngol 2004;68:1267-1272.

40) Yamamuro K, Ota T, Iida J, Nakanishi Y, Kishimoto N, Kishimoto T. Associations between the mismatch-negativity component and symptom severity in children and adolescents with attention deficit/hyperactivity disorder. Neuropsychiatr Dis Treat 2016;12:31833190.

41) Schreiber JE, Possin KL, Girard JM, Rey-Casserly C. Executive function in children with attention deficit/hyperactivity disorder: the NIH EXAMINER battery. J Int Neuropsychol Soc 2014;20:4151.

42) Auerbach RP, Admon R, Pizzagalli DA. Adolescent depression: stress and reward dysfunction. Harv Rev Psychiatry 2014;22:139148.

43) Barkley RA. Behavioral inhibition, sustained attention, and executive functions: constructing a unifying theory of ADHD. Psychol Bull 1997;121:65-94.

44) Geburek AJ, Rist F, Gediga G, Stroux D, Pedersen A. Electrophysiological indices of error monitoring in juvenile and adult attention deficit hyperactivity disorder (ADHD)--a meta-analytic appraisal. Int J Psychophysiol 2013;87:349-362.

45) Nigg JT. On inhibition/disinhibition in developmental psychopathology: views from cognitive and personality psychology and a working inhibition taxonomy. Psychol Bull 2000;126:220-246.

46) Nigg JT, Stavro G, Ettenhofer M, Hambrick DZ, Miller T, Henderson JM. Executive functions and ADHD in adults: evidence for selective effects on ADHD symptom domains. J Abnorm Psychol 2005;114:706-717.

47) Groom MJ, Cahill JD, Bates AT, Jackson GM, Calton TG, Liddle PF, et al. Electrophysiological indices of abnormal error-processing in adolescents with attention deficit hyperactivity disorder (ADHD). J Child Psychol Psychiatry 2010;51:66-76.

48) O'Connell RG, Bellgrove MA, Dockree PM, Lau A, Hester R, Garavan $\mathrm{H}$, et al. The neural correlates of deficient error awareness in attention-deficit hyperactivity disorder (ADHD). Neuropsychologia 2009;47:1149-1159.

49) Wiersema JR, van der Meere JJ, Roeyers H. ERP correlates of error monitoring in adult ADHD. J Neural Transm (Vienna) 2009;116: 371-379.

50) Herrmann MJ, Mader K, Schreppel T, Jacob C, Heine M, BoreattiHümmer A, et al. Neural correlates of performance monitoring in adult patients with attention deficit hyperactivity disorder (ADHD). World J Biol Psychiatry 2010;11:457-464.

51) Doehnert M, Brandeis D, Schneider G, Drechsler R, Steinhausen HC. A neurophysiological marker of impaired preparation in an 11-year follow-up study of attention-deficit/hyperactivity disorder (ADHD). J Child Psychol Psychiatry 2013;54:260-270.

52) Meyer A. A biomarker of anxiety in children and adolescents: a review focusing on the error-related negativity (ERN) and anxiety across development. Dev Cogn Neurosci 2017;27:58-68.

53) Carrasco M, Hong C, Nienhuis JK, Harbin SM, Fitzgerald KD, Gehring WJ, et al. Increased error-related brain activity in youth with obsessive-compulsive disorder and other anxiety disorders. Neurosci Lett 2013;541:214-218.

54) Meyer A, Hajcak G, Torpey DC, Kujawa A, Kim J, Bufferd S, et al. Increased error-related brain activity in six-year-old children with clinical anxiety. J Abnorm Child Psychol 2013;41:1257-1266.

55) Meyer A, Hajcak G, Torpey-Newman DC, Kujawa A, Klein DN. Enhanced error-related brain activity in children predicts the onset of anxiety disorders between the ages of 6 and 9. J Abnorm Psychol 2015;124:266-274. 
56) Lo SL, Schroder HS, Fisher ME, Durbin CE, Fitzgerald KD, Danovitch JH, et al. Associations between disorder-specific symptoms of anxiety and error-monitoring brain activity in young children. J Abnorm Child Psychol 2017;45:1439-1448.

57) Meyer A, Nelson B, Perlman G, Klein DN, Kotov R. A neural biomarker, the error-related negativity, predicts the first onset of generalized anxiety disorder in a large sample of adolescent females. J Child Psychol Psychiatry 2018;59:1162-1170.
58) Hajcak G, Franklin ME, Foa EB, Simons RF. Increased error-related brain activity in pediatric obsessive-compulsive disorder before and after treatment. Am J Psychiatry 2008;165:116-123.

59) Pizzagalli DA. Depression, stress, and anhedonia: toward a synthesis and integrated model. Annu Rev Clin Psychol 2014;10:393423.

60) Thapar A, Collishaw S, Pine DS, Thapar AK. Depression in adolescence. Lancet 2012;379:1056-1067. 